\title{
Metabolic (dysfunction) associated fatty liver disease: more evidence and a bright future
}

\author{
Ting-Yao Wang ${ }^{1}$, Jacob George ${ }^{2}$, Ming-Hua Zheng ${ }^{3,4,5}$ \\ ${ }^{1}$ Department of Nephrology, The First Affiliated Hospital of Wenzhou Medical University, Wenzhou, China; ${ }^{2}$ Storr Liver Centre, Westmead \\ Institute for Medical Research, Westmead Hospital and University of Sydney, Sydney, Australia; ${ }^{3}$ NAFLD Research Center, Department of \\ Hepatology, The First Affiliated Hospital of Wenzhou Medical University, Wenzhou, China; ${ }^{4}$ Institute of Hepatology, Wenzhou Medical University, \\ Wenzhou, China; ${ }^{5}$ Key Laboratory of Diagnosis and Treatment for the Development of Chronic Liver Disease in Zhejiang Province, Wenzhou, \\ China \\ Correspondence to: Ming-Hua Zheng, MD, PhD. NAFLD Research Center, Department of Hepatology, The First Affiliated Hospital of Wenzhou \\ Medical University, No. 2 Fuxue Lane, Wenzhou 325000, China. Email: zhengmh@wmu.edu.cn.
}

Submitted Aug 28, 2021. Accepted for publication Oct 11, 2021.

doi: $10.21037 / \mathrm{hbsn}-21-352$

View this article at: https://dx.doi.org/10.21037/hbsn-21-352

Recently, metabolic (dysfunction) associated fatty liver disease (MAFLD) has been proposed as a name to replace non-alcoholic fatty liver disease (NAFLD) (1). Controversy over the name is in full swing and evidence-based debate will determine the outcome. Evidence-based medicine is predicated on rigorous scientific data, clinical experience and patient preferences. So how does MAFLD stack up? We briefly summarize the existing evidence and find that MAFLD does hold up to its promise as an advance in the terminology for the disease we all treat.

Clinical applicability is the single most important criterion on which any new disease name should be judged. It includes aspects such as ease of use of the diagnostic criteria and utility in assessing natural history, hepatic, and extra-hepatic comorbidity. Based on current evidence (diagrammatically shown in Figure 1), MAFLD better identifies high-risk groups not only for liver-related outcomes but also for extrahepatic comorbidity (e.g., cardiovascular disease and chronic kidney disease). In these studies of the general population, the prevalence of MAFLD was $20.1-39.1 \%$ and the population with MAFLD is always greater than those with just NAFLD (2-7). Based on a Cohen's kappa of 0.76-0.92 (2-7), the concordance of the two definitions is high, implying that the new and positive definition may not impact disease prevalence data. However, what is critically different is that under the MAFLD label, specialists will be exclusively seeing the subset with fatty liver and metabolic dysregulation, the group most likely to develop hepatic and extra-hepatic adverse outcomes, while leaving the management of those with no metabolic dysregulation to primary care. At the population level, this has a huge impact on management. For example, in a country with a population of 1 billion, if $30 \%$ have MAFLD/NAFLD (300 million), 15 million (5\%) with NAFLD but not MAFLD could be managed in primary care. At the other end of the spectrum, those with dual etiology liver disease and MAFLD (e.g., HCV, HBV or alcohol use disorder) can be appropriately treated for their metabolic dysregulation in addition to their other liver disease.

Most studies indicate that MAFLD better identifies a higher clinical risk group, implicit as MAFLD selects for those with systemic metabolic dysregulation. However, some studies do not report this finding; one study (7) of 1,710 persons from the general population found that advanced liver fibrosis (defined as a median liver stiffness $\geq 9.7 \mathrm{KPa}$ ) was not different in MAFLD and NAFLD groups. Another study (8) of a population of 780 with liver biopsy reported that both groups have similar clinical characteristics and liver histology. Likewise, a cohort study (4) with a 7-year follow-up of $~ 900$ patients showed that MAFLD and NAFLD groups had similar new-onset cardiovascular events. Those excluded by the NAFLD definition but captured by the MAFLD definition however had higher baseline metabolic traits. All these results must be interpreted with caution given the small sample sizes 


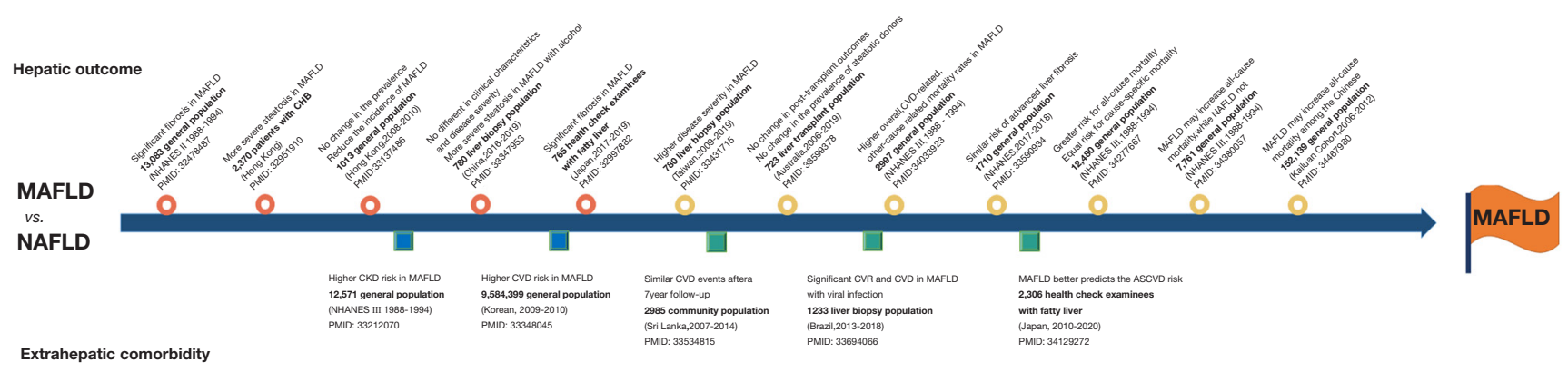

Figure 1 A summary of the latest studies on the comparison between metabolic (dysfunction) associated fatty liver disease (MAFLD) and non-alcoholic fatty liver disease (NAFLD).

as larger studies with up to 9 million patients uniformly indicate that MAFLD performs better in clinical practice (6).

We should also consider the impact of the new terminology on our understanding of the long-term outcomes of patients with NAFLD versus MAFLD. A majority of cross-sectional studies indicate that MAFLD presents with more severe liver histology than NAFLD, and some longitudinal studies find that MAFLD may have worse long-term outcomes. Nguyen et al. and Huang et al. analyzed data from the same database separately [i.e., the Third National Health and Nutrition Examination Survey (NHANES III) 1988-1994], and observed that MAFLD participants had higher all-cause mortality compared to those with NAFLD $(5,9)$. The latter study further showed that MAFLD and NAFLD had a similar risk of cardiovascular, cancer and diabetes-related mortality (9) implying that the MAFLD definition did not affect these outcomes. However, a large nationwide cohort study from Korea reported that MAFLD may have a greater risk of cardiovascular disease events than NAFLD (6). Considering that the definition of MAFLD implies the presence of metabolic dysregulation, but also allows for the coexistence of additional liver diseases such as alcohol-related liver disease or viral hepatitis infection which also affect mortality, it is reasonable to infer that MAFLD better captures a patient's mortality risk.

The new nomenclature not only impacts gastroenterologists, but also patients, nurses, other physicians, and drug developers. Some authors have surveyed the awareness of patients and medical practitioners and addressed the benefits of renaming from their perspective (10-12). The term "MAFLD" apparently increases disease awareness among patients, nurses, and physicians, and is thus better for patient care. Thus MAFLD is increasingly favored by stakeholders and accepted by patients. However, to date, a pharmaceutical perspective has not been forthcoming and is eagerly awaited.

More recently, MAFLD has entered a new phase with continuous additions to the evidence and widespread implementation to clinical practice. Eslam et al., have proposed multidisciplinary care of MAFLD and novel clinical trial design as the next steps to holistically improve the management of patients (13). Zheng et al. added to this with suggestions on MAFLD related cirrhosis care (14). The definition of MAFLD also brings us to "new" research directions. For instance, because metabolic syndrome is the key underpinning of MAFLD and the prevalence of MAFLD is as high as $50.7 \%$ among overweight or obese adults worldwide (15), it is reasonable to infer that other diseases closely related to metabolic syndrome or obesity (e.g., polycystic ovary syndrome and osteoarthritis) will also have a relationship. The non-invasive diagnosis based on NAFLD does not meet clinical needs (16), so it is worth studying whether current non-invasive scoring systems are suitable for MAFLD and for building consensus scoring systems.

The Asian-Pacific Association for the Study of the Liver (APASL) (1), the Chinese Society of Hepatology (CSH) (17), the Latin American Association for the Study of Liver (ALEH) (18), the Middle East and North Africa Consensus (19), as well as sub-Saharan Africa (20), have accepted the renaming to MAFLD. However, the European Association for the Study of the Liver (EASL) and the American Association for the Study of Liver Diseases (AASLD) have not released a position statement. What can be said however is that the concept increasingly resonates with clinicians, health care providers and patients and as we speak, "MAFLD" usage is evolving towards a new equilibrium. 


\section{Acknowledgments}

Funding: This work was supported by grants from the National Natural Science Foundation of China (82070588), High Level Creative Talents from Department of Public Health in Zhejiang Province (S2032102600032) and Project of New Century 551 Talent Nurturing in Wenzhou.

\section{Footnote}

Provenance and Peer Review: This article was commissioned by the editorial office, Hepatobiliary Surgery and Nutrition. The article did not undergo external peer review.

Conflicts of Interest: All authors have completed the ICMJE uniform disclosure form (available at https://hbsn. amegroups.com/article/view/10.21037/hbsn-21-352/coif). Dr. MHZ serves as an unpaid editorial board member of Hepatobiliary Surgery and Nutrition. The other authors have no conflicts of interest to declare.

Ethical Statement: The authors are accountable for all aspects of the work in ensuring that questions related to the accuracy or integrity of any part of the work are appropriately investigated and resolved.

Open Access Statement: This is an Open Access article distributed in accordance with the Creative Commons Attribution-NonCommercial-NoDerivs 4.0 International License (CC BY-NC-ND 4.0), which permits the noncommercial replication and distribution of the article with the strict proviso that no changes or edits are made and the original work is properly cited (including links to both the formal publication through the relevant DOI and the license). See: https://creativecommons.org/licenses/by-nc-nd/4.0/.

\section{References}

1. Eslam M, Sarin SK, Wong VW, et al. The Asian Pacific Association for the Study of the Liver clinical practice guidelines for the diagnosis and management of metabolic associated fatty liver disease. Hepatol Int 2020;14:889-919.

2. Wong VW, Wong GL, Woo J, et al. Impact of the New Definition of Metabolic Associated Fatty Liver Disease on the Epidemiology of the Disease. Clin Gastroenterol Hepatol 2021;19:2161-2171.e5.

3. Sun DQ, Jin Y, Wang TY, et al. MAFLD and risk of CKD. Metabolism 2021;115:154433.
4. Niriella MA, Ediriweera DS, Kasturiratne A, et al. Outcomes of NAFLD and MAFLD: Results from a community-based, prospective cohort study. PLoS One 2021;16:e245762.

5. Nguyen VH, Le MH, Cheung RC, et al. Differential Clinical Characteristics and Mortality Outcomes in Persons With NAFLD and/or MAFLD. Clin Gastroenterol Hepatol 2021;19:2172-81.e6.

6. Lee H, Lee YH, Kim SU, et al. Metabolic DysfunctionAssociated Fatty Liver Disease and Incident Cardiovascular Disease Risk: A Nationwide Cohort Study. Clin Gastroenterol Hepatol 2021;19:2138-2147.e10.

7. Ciardullo S, Perseghin G. Prevalence of NAFLD, MAFLD and associated advanced fibrosis in the contemporary United States population. Liver Int 2021;41:1290-3.

8. Zheng KI, Sun DQ, Jin Y, et al. Clinical utility of the MAFLD definition. J Hepatol 2021;74:989-91.

9. Huang Q, Zou X, Wen X, et al. NAFLD or MAFLD: Which Has Closer Association With All-Cause and CauseSpecific Mortality?-Results From NHANES III. Front Med (Lausanne) 2021;8:693507.

10. Clayton M, Fabrellas N, Luo J, et al. From NAFLD to MAFLD: Nurse and allied health perspective. Liver Int 2021;41:683-91.

11. Fouad Y, Gomaa A, Semida N, et al. Change from NAFLD to MAFLD increases the awareness of fatty liver disease in primary care physicians and specialists. J Hepatol 2021;74:1254-6.

12. Shiha G, Korenjak M, Eskridge W, et al. Redefining fatty liver disease: an international patient perspective. Lancet Gastroenterol Hepatol 2021;6:73-9.

13. Eslam M, Ahmed A, Després JP, et al. Incorporating fatty liver disease in multidisciplinary care and novel clinical trial designs for patients with metabolic diseases. Lancet Gastroenterol Hepatol 2021;6:743-53.

14. Zheng KI, Eslam M, George J, et al. When a new definition overhauls perceptions of MAFLD related cirrhosis care. Hepatobiliary Surg Nutr 2020;9:801-4.

15. Liu J, Ayada I, Zhang X, et al. Estimating Global Prevalence of Metabolic Dysfunction-Associated Fatty Liver Disease in Overweight or Obese Adults. Clin Gastroenterol Hepatol 2021. doi: 10.1016/ j.cgh.2021.02.030. [Epub ahead of print].

16. Zhou YJ, Zheng KI, Targher G, et al. Non-invasive diagnosis of non-alcoholic steatohepatitis and liver fibrosis. Lancet Gastroenterol Hepatol 2021;6:9-10.

17. Nan Y, An J, Bao J, et al. The Chinese Society of Hepatology position statement on the redefinition of fatty 
liver disease. J Hepatol 2021;75:454-61.

18. Mendez-Sanchez N, Arrese M, Gadano A, et al. The Latin American Association for the Study of the Liver (ALEH) position statement on the redefinition of fatty liver disease. Lancet Gastroenterol Hepatol 2021;6:65-72.

19. Shiha G, Alswat K, Al Khatry M, et al. Nomenclature and definition of metabolic-associated fatty liver disease: a consensus from the Middle East and north Africa. Lancet Gastroenterol Hepatol 2021;6:57-64.

20. Spearman CW, Desalegn H, Ocama P, et al. The subSaharan Africa position statement on the redefinition of fatty liver disease: From NAFLD to MAFLD. J Hepatol 2021;74:1256-8.

Cite this article as: Wang TY, George J, Zheng MH. Metabolic (dysfunction) associated fatty liver disease: more evidence and a bright future. HepatoBiliary Surg Nutr 2021;10(6):849-852. doi: 10.21037/hbsn-21-352 